OPEN ACCESS

Edited by:

Marek Marzec,

University of Silesia of Katowice,

Poland

Reviewed by:

Weiqiang Li,

RIKEN, Japan

Kosuke Fukui,

Okayama University of Science,

Japan

*Correspondence:

Mikihisa Umehara

umehara@toyo.jp

Specialty section:

This article was submitted to

Plant Physiology,

a section of the journal

Frontiers in Plant Science

Received: 23 October 2019

Accepted: 29 January 2020

Published: 25 February 2020

Citation:

Shindo M, Yamamoto S, Shimomura K and Umehara M (2020) Strigolactones

Decrease Leaf Angle in Response to Nutrient Deficiencies in Rice.

Front. Plant Sci. 11:135.

doi: 10.3389/fpls.2020.00135

\section{Strigolactones Decrease Leaf Angle in Response to Nutrient Deficiencies in Rice}

\author{
Masato Shindo ${ }^{1}$, Shu Yamamoto ${ }^{2}$, Koichiro Shimomura ${ }^{1}$ and Mikihisa Umehara ${ }^{1,2 *}$ \\ ${ }^{1}$ Graduate School of Life Sciences, Toyo University, Ora-gun, Japan, ${ }^{2}$ Department of Applied Biosciences, Toyo University, \\ Ora-gun, Japan
}

Strigolactones (SLS) are a class of plant hormones that are synthesized from $\beta$-carotene through sequential reactions catalyzed by DWARF (D) 27, D17, D10, and OsMORE AXILLARY GROWTH (MAX) 1 in rice (Oryza sativa L.). In rice, endogenous SL levels increase in response to deficiency of nitrogen, phosphate, or sulfate $(-\mathrm{N},-\mathrm{P}$, or $-\mathrm{S})$. Rice SL mutants show increased lamina joint (LJ) angle as well as dwarfism, delayed leaf senescence, and enhanced shoot branching. The LJ angle is an important trait that determines plant architecture. To evaluate the effect of endogenous SLs on LJ angle in rice, we measured LJ angle and analyzed the expression of SL-biosynthesis genes under macronutrient deficiencies. In the "Shiokari" background, LJ angle was significantly larger in SL mutants than in the wild-type (WT). In WT and SL-biosynthesis mutants, direct treatment with the SL synthetic analog GR24 decreased the LJ angle. In WT, deficiency of $\mathrm{N}, \mathrm{P}$, or S, but not of $\mathrm{K}, \mathrm{Ca}, \mathrm{Mg}$, or Fe decreased LJ angle. In SL mutants, deficiency of $\mathrm{N}$, $\mathrm{P}$, or $\mathrm{S}$ had no such effect. We analyzed the time course of SL-related gene expression in the LJ of WT deficient in N, P, or S, and found that expression of SL-biosynthesis genes increased 2 or 3 days after the onset of deficiency. Expression levels of both the SLbiosynthesis and signaling genes was particularly strongly increased under -P. Rice cultivars "Nipponbare", "Norin 8", and "Kasalath" had larger LJ angle than "Shiokari", interestingly with no significant differences between WT and SL mutants. In "Nipponbare", endogenous SL levels increased and the LJ angle was decreased under $-\mathrm{N}$ and $-\mathrm{P}$. These results indicate that SL levels increased in response to nutrient deficiencies, and that elevated endogenous SLs might negatively regulate leaf angle in rice.

Keywords: lamina joint, nitrogen deficiency, Oryza sativa, phosphate deficiency, strigolactone, sulfur deficiency

\section{INTRODUCTION}

Leaf angle is generally defined as the inclination between the leaf blade midrib and the stem, and is one of the most important plant architecture parameters that influence light interception, photosynthetic efficiency, and planting density (Mantilla-Perez and Salas Fernandez, 2017). Leaf erectness enhance light capture, improve photosynthetic assimilation, and help high density planting (Sinclair and Sheehy, 1999; Sakamoto et al., 2006). Thus, leaf angle is an important agricultural trait that contributes to grain yield in cereal crops. In rice (Oryza sativa L.), leaf angle is 
determined by the shape of the lamina joint (LJ), which connects the leaf blade and sheath (Hoshikawa, 1989). The lack of cell longitudinal elongation results in a small LJ angle, and cell elongation on the adaxial side of the LJ induces leaf blade bending away from vertical axis to a more horizontal position (Cao and Chen, 1995; Zhang et al., 2009; Zhao et al., 2010).

Rice LJ angle is regulated by plant hormones. Brassinosteroids (BR) stimulate elongation of adaxial parenchyma cells at the LJ and increase LJ angle (Wada et al., 1981; Cao and Chen, 1995; Zhang et al., 2009). BR also inhibit proliferation of abaxial sclerenchyma cells by controlling U-type cyclin CYC U4;1 (Sun et al., 2015). Gibberellin stimulates cell elongation, and interacts with BR signaling by several regulators (Shimada et al., 2006; Wang et al., 2009). Gibberellin reduced the leaf angle by inhibiting $\mathrm{BR}$ response, demonstrating that gibberellin is a negative regulator of lamina inclination (Tong et al., 2014). Auxin also increases LJ angle via a BRI1-dependent and -independent pathway (Nakamura et al., 2009; Zhao et al., 2013; Zhang et al., 2015). In contrast, methyl jasmonate represses BR biosynthesis and signaling, and reduces LJ angle (Gan et al., 2015). Recently, it was reported that leaf inclination is higher in strigolactone (SL) mutants than in wild type (WT), and that exogenously applied GR24, a synthetic SL analog, decreases the large inclination in SL-biosynthesis mutants (Li et al., 2014). However, how LJ angle is regulated by SL signal remains unknown.

SL was originally isolated from plant root exudates as a seed germination stimulant of witchweed (Cook et al., 1966; Cook et al., 1972). Later, SL was identified as a stimulator of hyphal branching in arbuscular mycorrhizal fungi, which supply soil nutrients to host plants (Akiyama et al., 2005). The previous studies proposed that SL functions as a communication signal for parasitism and symbiosis in the rhizosphere (Bouwmeester et al., 2007). More later, SLs were found as a class of phytohormones that inhibit shoot branching (Gomez-Roldan et al., 2008; Umehara et al., 2008). In this time, SLs are also known to control root architecture and promote leaf senescence, endosperm development, and secondary growth (Agusti et al., 2011; Kapulnik et al., 2011; Ruyter-Spira et al., 2011; Yamada et al., 2014; Ueda and Kusaba, 2015; Yamada et al., 2018).

In rice, SL-biosynthesis mutants $d 27, d 17 /$ high tillering $d w a r f$ ( $h t d) 1$, and $d 10$ are well characterized. D27, D17/HTD1, and D10 encode $\beta$-carotene isomerase, carotenoid cleavage dioxygenase (CCD) 7, and CCD8, respectively (Zou et al., 2006; Arite et al., 2007; Lin et al., 2009). In rice SL biosynthesis, all-trans- $\beta$ carotene is converted to carlactone by sequential reactions via D27, D17, and D10 (Alder et al., 2012; Seto et al., 2014); carlactone is converted to SL by MAX1, which is a cytochrome P450 CYP711 family protein (Zhang et al., 2014). There are five MAX1 homologs in rice (Nelson et al., 2004); among them, Os900 converts carlactone to 4-deoxyprobanchol via carlactonoic acid, and Os1400 converts carlactone to 4deoxyprobanchol (4DO), and 4DO to orobanchol (Yoneyama et al., 2018a). The SL signaling mutant $d 14$ has a mutation in an $\alpha / \beta$-hydrolase protein (Arite et al., 2009). D14 protein has a dual function as a receptor and deactivator of bioactive SLs (Seto et al.,
2019). Another SL signaling mutant, $d 3$, has a mutation in leucine-rich-repeat F-box protein (Ishikawa et al., 2005), which acts as a recognition subunit in the SKP-CUL1-F-box (SCF) protein complex, binds target proteins and directs them for proteasomal degradation. The SL-insensitive mutant $d 53$ has a mutation in a repressor of SL signaling (Jiang et al., 2013; Zhou et al., 2013). D53 is the target protein of the $\mathrm{SCF}^{\mathrm{D} 3}$ ubiquitination complex in SL signaling.

SLs are produced mainly in roots in response to nitrogen and/ or phosphate deficiencies in several plant species (Yoneyama et al., 2007a; Yoneyama et al., 2007b; Lopez-Raez et al., 2008; Yoneyama et al., 2012). In a rice cultivar "Shiokari", SLs are produced in response to nitrogen, phosphate, and sulfur deficiencies (Umehara et al., 2010; Sun et al., 2014; Shindo et al., 2018). In this study, we investigated the effect of nutrient deficiencies on endogenous SL production and on LJ angle in rice. Furthermore, we compared the LJ angles of WT and $d$ mutants grown under nutrient-deficient or sufficient conditions, analyzed SL-related gene expression, and measured canonical SL levels in roots.

\section{MATERIALS AND METHODS}

\section{Plant Growth Conditions and Measurement of LJ Angle}

In this study, we used wild-type (WT) plants and $d$ warf mutants $d 27-1, d 17-1, d 10-1, d 14-1$, and $d 3-1$ in the "Shiokari" background, $d 10-2, d 17-2$ in the "Nipponbare" background, and $d 53$ in the "Norin 8" background (Ishikawa et al., 2005; Arite et al., 2007; Umehara et al., 2008; Arite et al., 2009; Lin et al., 2009; Zhou et al., 2013; Kobae et al., 2018). The seeds were kindly provided by Prof. Junko Kyozuka (Tohoku University) and by Dr. Hiroaki Iwai and were propagated in a glass room at the Research Center for Life and Environmental Sciences, Toyo University, Japan. Rice seedlings were grown hydroponically as described previously (Umehara et al., 2008). Surface-sterilized seeds were incubated in sterile water at $25^{\circ} \mathrm{C}$ in the dark for 1 day, and germinated seeds were transferred to hydroponic culture medium (Kamachi et al., 1991) solidified with $0.6 \%$ agar and cultured under $16 \mathrm{~h}$ fluorescent white light (130-180 $\mu \mathrm{mol} \mathrm{m} \mathrm{m}^{-2} \mathrm{~s}^{-1}$ ) at $25^{\circ} \mathrm{C}$ and $8 \mathrm{~h}$ dark at $23^{\circ} \mathrm{C}$ for 6 days (preculture). Seedlings of similar size were then transferred to hydroponic medium containing $1 \mathrm{mM} 2$-( $N$-morpholino) ethanesulfonic acid (MES) buffer ( $\mathrm{pH}$ 5.7) for a further 3, 6, or 24 days. Seedlings were placed in soil and cultivated in a glass room for 34 days. LJ angle (between leaf blade and sheath) of the 2nd, 3rd, and 4th leaves was measured on leaf images in ImageJ software v. 1.50 (Schneider et al., 2012).

\section{Chemicals}

rac-GR24, a synthetic SL analog, was purchased from Chiralix (Nijmegen, The Netherlands) and dissolved in acetone. Mock solution or $20 \mu \mathrm{M}$ rac-GR24 containing $0.1 \%$ Tween-20 were spotted $(2 \mu \mathrm{l})$ onto the LJ of WT and $d$ mutants on days 0 and 3 of cultivation in hydroponic medium. The following standards 
were used for SL quantification: $4 \mathrm{DO}, d_{1}$-labeled $4 \mathrm{DO}, d_{3}$-labeled $4 \mathrm{DO}$, orobanchol, $d_{1}$-labeled orobanchol, and $d_{3}$-labeled orobanchol; all were kindly provided by Prof. Kohki Akiyama (Osaka Prefecture University).

\section{RNA Extraction and qRT-PCR}

Total RNA was extracted from approximately $25 \mathrm{mg}$ LJ segments using an RNeasy Plant mini kit (Qiagen, Hilden, Germany) following the instructions in the user manual. We used $0.1 \mu \mathrm{g}$ of the total RNA for cDNA synthesis with a ReverTra Ace qPCR RT kit for quantitative real-time PCR (qRT-PCR) (Toyobo, Osaka, Japan). qRT-PCR was performed in a StepOnePlus thermocycler (Thermo Fisher Scientific, Waltham, MA, USA) with a Thunderbird Probe qPCR mix (Toyobo). Expression of ubiquitin was used as an internal standard. Expression levels of SL-related genes were quantified using the specific primers and probes used in a previous study (Shindo et al., 2018).

\section{SL Purification and Quantification}

We measured the levels of $4 \mathrm{DO}$ and orobanchol in roots as described previously (Hasegawa et al., 2018; Shindo et al., 2018) with some modifications. Germinated seeds were pre-cultured in agar culture media for 7 days and the seedlings were grown in hydroponic culture media for a further 7 days. Roots (ca. $1 \mathrm{~g}$ ) of 14-day-old seedlings were homogenized in $10 \mathrm{ml}$ acetone containing $100 \mathrm{pg}$ each of $d_{1}$-labeled $4 \mathrm{DO}$ and orobanchol as internal standards; the homogenates were filtered with Bond Elute reservoirs (Agilent, Santa Clara, CA, USA) and evaporated to dryness under nitrogen gas. The extracts were dissolved in 4 ml water adjusted to $\mathrm{pH} 2-3$ with $1 \mathrm{~N} \mathrm{HCl}$ and extracted twice with $4 \mathrm{ml}$ ethyl acetate. The ethyl acetate phase was evaporated to dryness under nitrogen gas. The extracts were dissolved in $10 \%$ acetone, loaded onto Oasis HLB 3-ml cartridges (Waters), washed with $10 \%$ acetone, and eluted with $60 \%$ acetone. The eluates were dissolved in ethyl acetate: $n$-hexane (15:85) and loaded onto Sep-Pak Silica 1-ml cartridges (Waters). After the cartridges were washed with ethyl acetate: $n$-hexane (15:85), $4 \mathrm{DO}$ was eluted with ethyl acetate: $n$-hexane (35:65) and orobanchol with ethyl acetate: $n$-hexane (50:50). LJ sample (ca. $0.15 \mathrm{~g}$ ) were homogenized in $10 \mathrm{ml}$ acetone containing $100 \mathrm{pg}$ each of $d_{3}$-labeled $4 \mathrm{DO}$ and orobanchol; the homogenates were filtered with Bond Elute reservoirs (Agilent, City CA, USA) and evaporated to dryness under nitrogen gas. The extracts were dissolved in $4 \mathrm{ml} \mathrm{50 \%}$ acetonitrile adjusted to $\mathrm{pH} 2-3$ with $1 \mathrm{~N}$ $\mathrm{HCl}$, and $4 \mathrm{ml}$ hexane, mixed gently, centrifuged at 3,400 rpm for $5 \mathrm{~min}$, and the hexane-phase was removed. After the aqueousphase was extracted with $4 \mathrm{ml}$ ethyl acetate twice, 4DO, and orobanchol in the ethyl acetate-phase were purified according to the method in roots.

Purified SL-containing fractions were dissolved in 50\% acetonitrile and subjected to liquid chromatography-tandem mass spectrometry (LC-MS/MS) analysis using a system consisting of a quadrupole tandem mass spectrometer (3200 QTRAP; Sciex, Framingham, MA, USA) and a high-performance liquid chromatograph (Prominence, Shimadzu, Kyoto, Japan) equipped with a reverse-phase column (Acquity UPLC BEH-
C18, $2.1 \times 50 \mathrm{~mm}, 1.7 \mu \mathrm{m}$, Waters). Previous papers describe LC and MS parameters for 4DO (Shindo et al., 2018) and orobanchol (Hasegawa et al., 2018) analysis. Data were analyzed in Analyst 1.5.1 and Multi Quant 2.0.2 (Sciex) software.

\section{Statistical Analysis}

Statistical analysis was performed in SPSS 24 software (IBM SPSS Inc., Armonk, NY USA). Student's $t$-test $(P<0.05)$ was used for pairwise comparisons and Tukey's honestly significant difference (HSD) $(P<0.05)$ for multiple comparisons.

\section{RESULTS}

\section{LJ angle in Rice SL Mutants in "Shiokari" Background}

Many SL-biosynthesis and signaling mutants are available in the background of the rice cultivar 'Shiokari'. The 2nd, 3rd, and 4th leaves of the seedlings that had been grown hydroponically for a month are shown in Figure 1A. The LJ angle of SL mutants was almost twice as large as that of WT 'Shiokari' (Figure 1B). In seedlings grown in soil for 40 days, it was slightly smaller, but that of SL mutants was also almost twice as large as that of WT (Supplementary Figure S1), indicating that the LJ angle of SL mutants was increased regardless of the growth conditions. To simplify the comparison between WT and SL mutants, we focused on the 2nd LJ angle. After pre-culture for 6 days (Figure 2A), the 2 nd LJ angle was $0^{\circ}$, with no significant difference between WT and SL mutants (Figure 2B). However, it became larger in SL mutants than in WT on day 7, continued to increase from day 8 to day 11 , and reached maximum in both WT and SL mutants on day 12 (Figure 2B). In a previous study (Li et al., 2014), the LJ angle was greater in $d 3-1$ than in other $d$ mutants, but in our experimental conditions, it was smaller in $d 3-1$ (Figure 2B). We confirmed that exogenously applied $20 \mu \mathrm{M}$ GR24 (SL synthetic analog) strongly decreased LJ angle in WT and the SL-biosynthesis mutants $d 10-1, d 17-1$, and $d 27-1$, but had no effect on the SL-signaling mutants $d 3-1$ and $d 14-1$ (Supplementary Figure S2).

\section{Effects of Macronutrient Deficiencies on LJ Angle, SL-Related Gene Expression, and SL Levels in the "Shiokari" Background}

In "Shiokari", SL production in roots is stimulated in response to deficiency of nitrogen, phosphate, or sulfate (Shindo et al., 2018). To evaluate the effects of macronutrient deficiencies on LJ angle, we grew WT and SL mutant seedlings under deficiencies of nitrogen, phosphate, sulfate, potassium, calcium, or magnesium $(-\mathrm{N},-\mathrm{P},-\mathrm{S},-\mathrm{K},-\mathrm{Ca},-\mathrm{Mg}$, and $-\mathrm{Fe})$. The LJ angle of WT decreased by almost half under $-\mathrm{N},-\mathrm{P}$, and $-\mathrm{S}$ in comparison with the control, whereas that of SL mutants was not affected by a deficiency of any of these macronutrients (Figures 3A, B). Using LC-MS/MS, we analyzed the levels of canonical SLs, 4DO and orobanchol, in roots. The 4DO levels in roots increased under $-\mathrm{N},-\mathrm{P}$, and $-\mathrm{S}$, but the levels of orobanchol were below the detection limit in "Shiokari" (Figure 3C). 


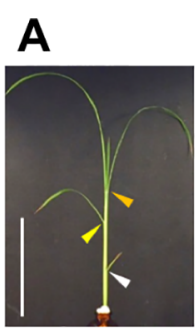

Shiokari

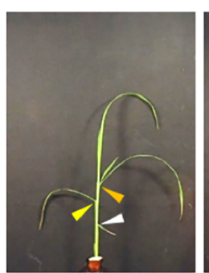

d10-1

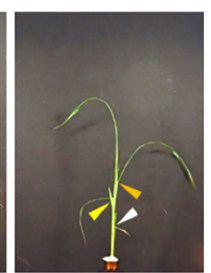

$d 17-1$

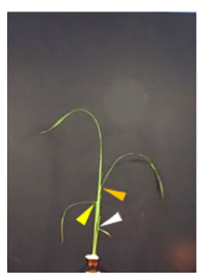

$d 27-1$

SL-biosynthesis mutants

B

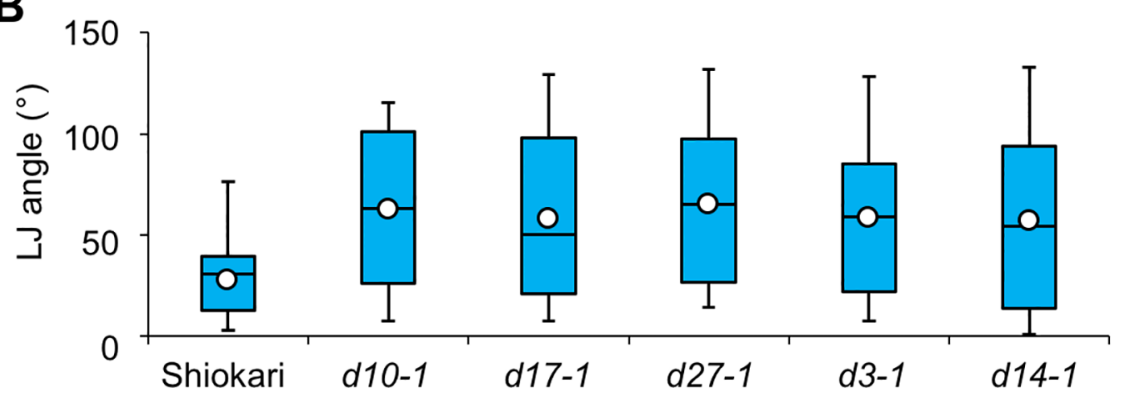

FIGURE 1 | Lamina joint (LJ) angle in "Shiokari"-background wild-type and strigolactone (SL) mutant rice seedlings. (A) Images of 30-d-old seedlings. Arrowheads: white, 2nd LJ; yellow, 3rd LJ; orange, 4th LJ. Bar: $10 \mathrm{~cm}$. (B) LJ angle measured with ImageJ software. White circles indicate average ( $n=60)$.

A
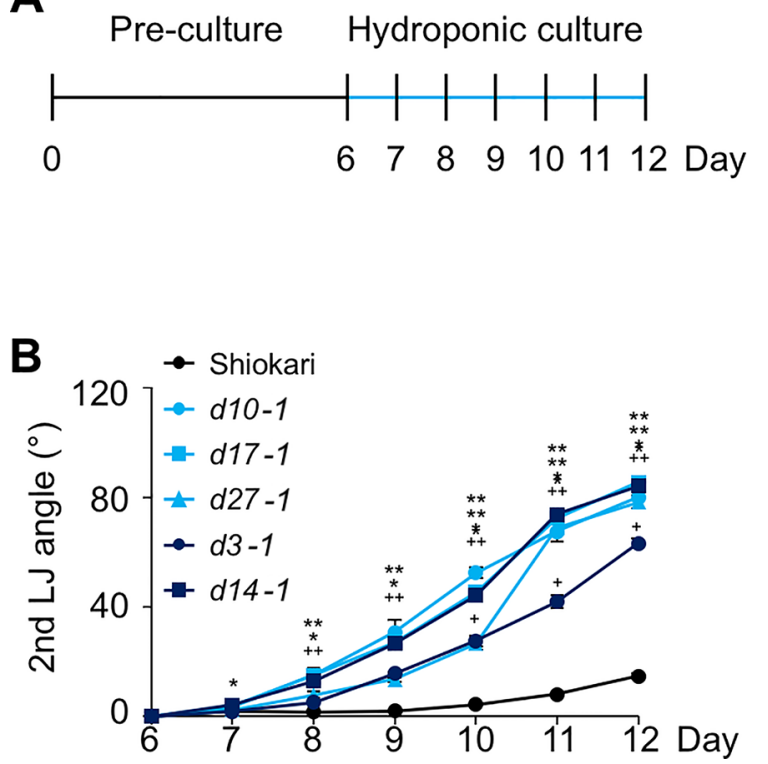

FIGURE 2 | Time-course analysis of $L J$ angle in the 2nd leaf of rice seedlings. (A) Timeline of the experiment. After pre-culture, seedlings were transferred to nutrient-sufficient hydroponic medium. (B) LJ angle of wild-type "Shiokari" and SL mutants. Asterisks and plus signs indicate significant differences in ANOVA (all $P<0.05)$ vs. 'Shiokari'. Data are means \pm S.E. $(n=5)$.
To explore whether SL levels increase in LJ under $-\mathrm{N},-\mathrm{P}$, and $-\mathrm{S}$, we analyzed the expression levels of SL-biosynthesis and signaling genes on days 7,8 , and 9 , i.e. before a large increase in LJ angle (Figure 4A). On day 7, the expression of OsMAX1 decreased under $-\mathrm{S}$ (Figure 4B). On day 8, the expression of all SL-biosynthesis genes under $-\mathrm{P}$ increased, whereas that of OsMAX1 under $-S$ returned to the control levels (Figure 4B). On day 9, the expression of some SL-biosynthesis genes increased under $-\mathrm{N},-\mathrm{S}$, and $-\mathrm{P}$ (Figure 4B). All SLbiosynthetic genes we analyzed were up-regulated in 8-day-old seedlings grown under $-\mathrm{P}$. Thus, we tried to analyze the levels of canonical SLs, 4DO and orobanchol, in LJ of the seedlings. However, we were unable to detect $4 \mathrm{DO}$ or orobanchol in $\mathrm{LJ}$ (Supplementary Figure S3). Among SL-signaling genes, the expression of D3 and D14 also increased under $-\mathrm{P}$ on days 8 and 9 (Figure 5). Expression of these genes slightly but significantly decreased under $-\mathrm{N}$ on day 8 .

\section{LJ Angle and Response of Nutrient Deficiencies in Different Rice Cultivars}

The LJ angle was smaller in "Shiokari" than in "Nipponbare", "Norin 8", and "Kasalath" (Supplementary Figures S4A, B). It did not differ significantly between WT "Nipponbare" and the d10-2 and d17-2 mutants in the "Nipponbare" background (Supplementary Figure S4C), or between WT "Norin 8" and $d 53$ in the "Norin 8" background (Supplementary Figure S4D). In WT "Nipponbare", it decreased by half under $-\mathrm{N}$ and $-\mathrm{P}$, but 

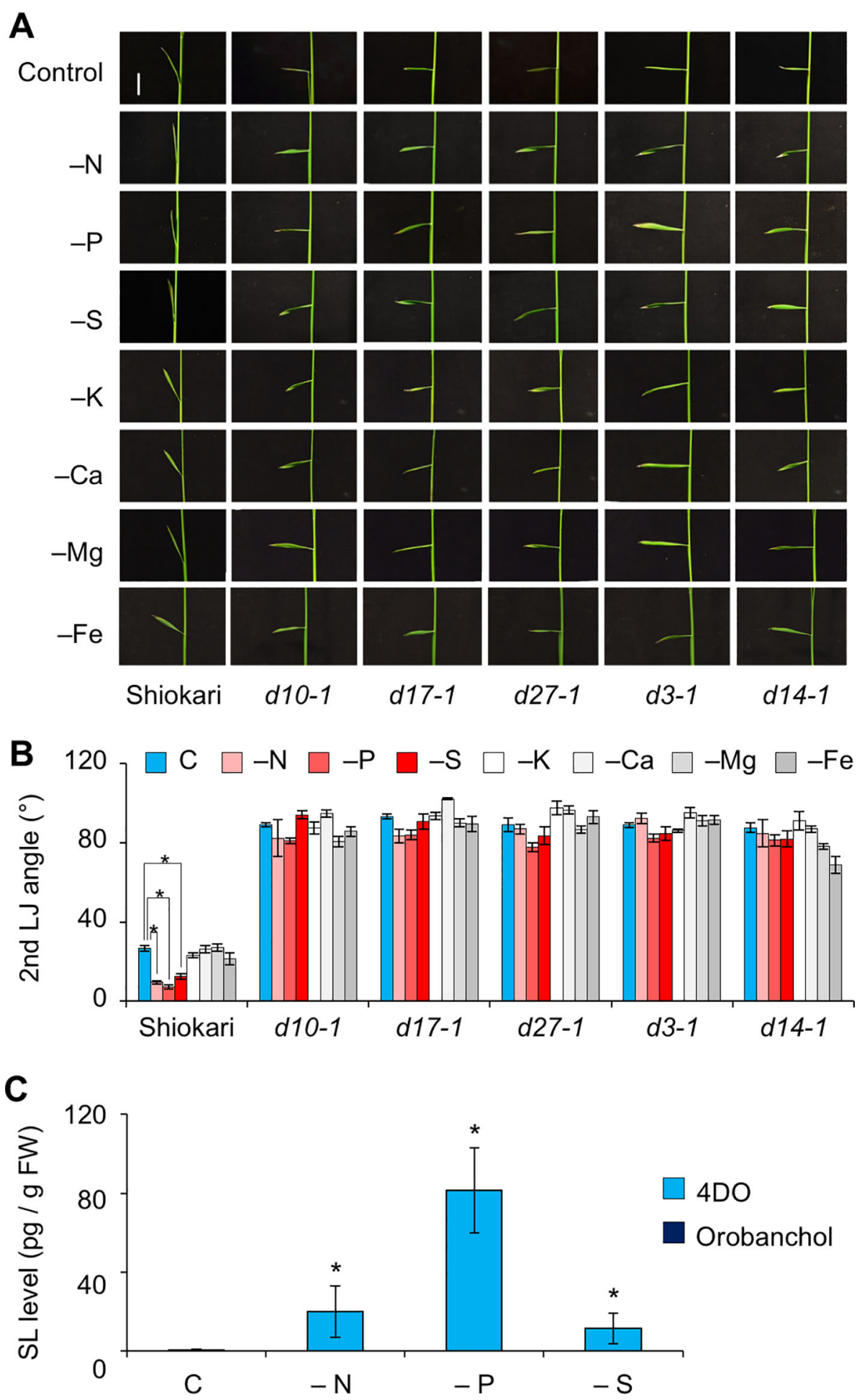

FIGURE 3 | Effect of macronutrient deficiency on LJ angle and SL levels in "Shiokari"-background seedlings. (A) Images of the 2nd leaves of 12-d-old seedlings. Bar, $1 \mathrm{~cm}$. (B) Second-LJ angle under macronutrient deficiencies. C, control. ${ }^{\star} P<0.05$ (Student's $t$-test). Data are means \pm S.E. ( $n=5$; eight seedlings per experiment). (C) SL levels in roots under nitrogen, phosphate, or sulfate deficiency in roots of 14 -d-old seedlings. C, control. ${ }^{\star} P<0.05$ (Student's $t$-test vs. control). Data are means \pm S.E. $(n=4)$. 
A

Pre-culture Hydroponic culture

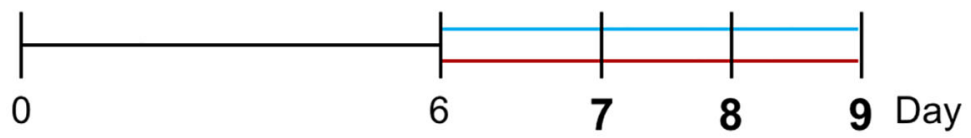

Control (C)

Nutrient

deficiency

B

Day 7

Day 8

Day 9
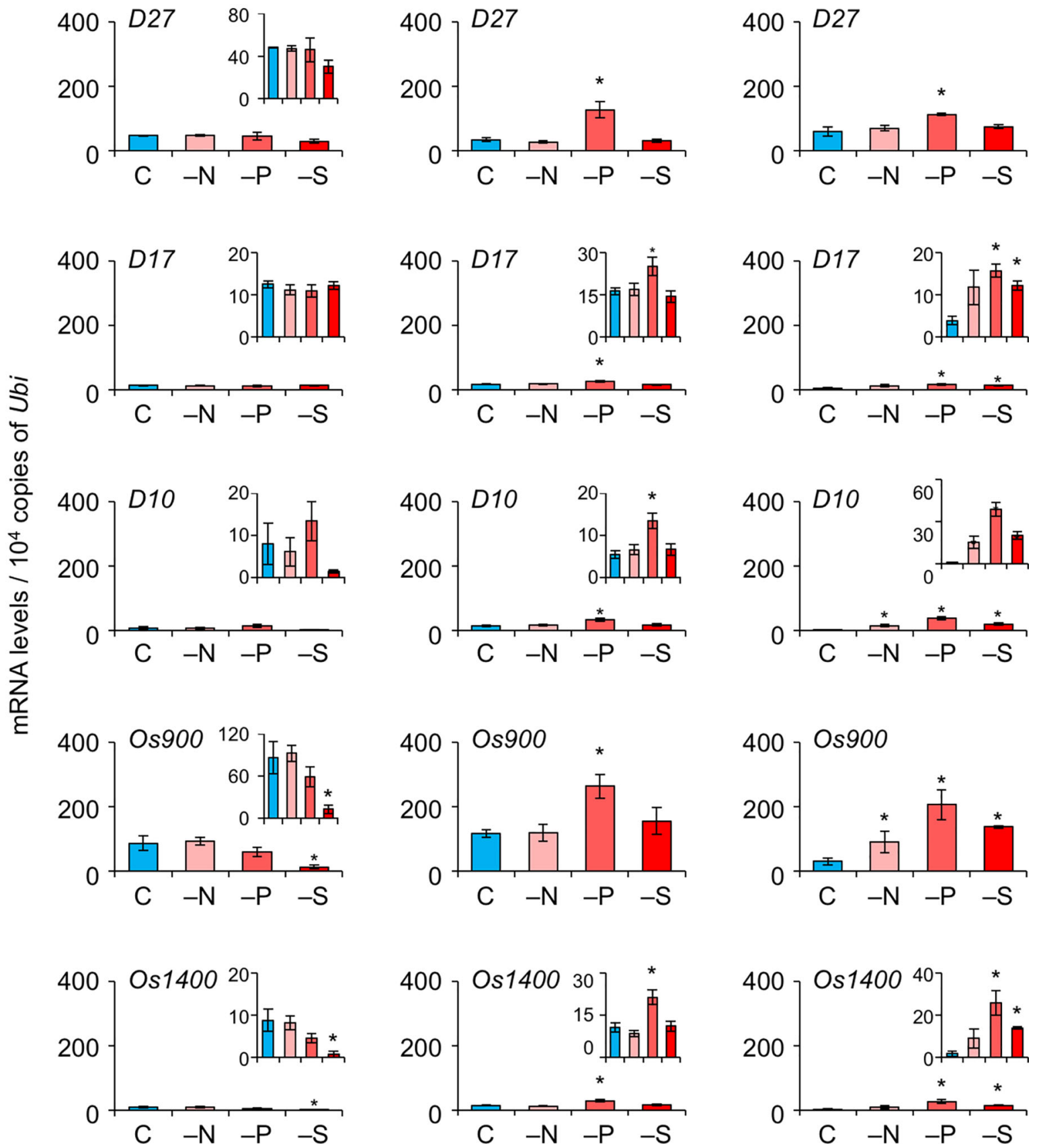

FIGURE 4 | Expression of SL-biosynthesis genes in LJ under nitrogen, phosphate, or sulfate deficient condition. (A) Timeline of the experiment. After pre-culture, seedlings were transferred to control or nutrient-deficient hydroponic medium. Gene expression was analyzed on days 7, 8, and 9. (B) Transcript levels in the 2nd LJ. C, control. ${ }^{*} P<0.05$ (Student's $t$-test vs. control). Data are means \pm S.E. $(n=3$, six seedlings per experiment). 


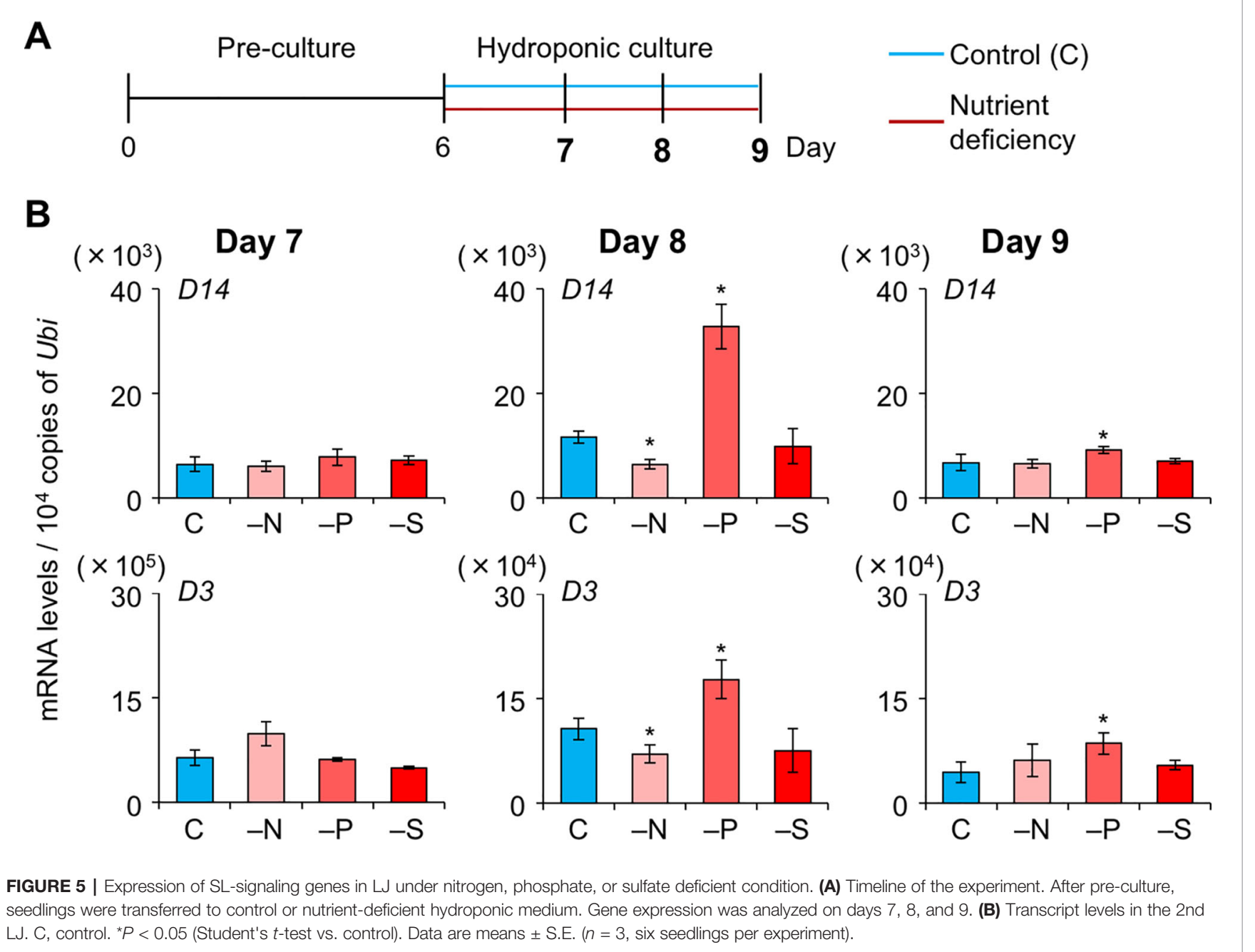

did not change under $-S$ (Figures 6A, B). That of $d 17-2$ did not change under $-\mathrm{N},-\mathrm{P}$, or $-\mathrm{S}$ (Figures $6 \mathbf{A}, \mathbf{B}$ ). In "Nipponbare" roots, 4DO levels increased under $-\mathrm{N}$ and especially $-\mathrm{P}$ and orobanchol was only detected under $-\mathrm{P}$ (Figure 6C). Unlike in "Shiokari" (Figure 3C), we detected no significant SL increase under $-\mathrm{S}$ in "Nipponbare". Exogenously applied $20 \mu \mathrm{M}$ GR24 decreased LJ angle in WT and the SL-biosynthesis mutants $d 17-2$ (Supplementary Figure S5).

\section{DISCUSSION}

We investigated the effects of SLs on LJ angle under macronutrient deficiencies in rice. In response to $-\mathrm{N},-\mathrm{P}$, and $-\mathrm{S}$, SL-biosynthesis genes were upregulated and endogenous SL levels increased, decreasing LJ angle in "Shiokari" (Figures 3, 4, and 7). Phosphate deficiency had the strongest effect on expression of SL-biosynthesis genes in LJ and SL production in roots (Figures 3 and 4). The results are consistent with our previous report that SL levels increase in response to $-\mathrm{N},-\mathrm{P}$, and
-S in "Shiokari" (Shindo et al., 2018). Expression of SLbiosynthesis genes in $-\mathrm{P}$ were higher than that in $-\mathrm{N}$ and $-\mathrm{S}$, but the LJ angle of rice seedlings under $-\mathrm{N}$ and $-\mathrm{S}$ is narrow at the same level as that of -P plants (Figures 3 and 4). Expression of SL-signaling genes, D3 and D14, were higher than that of SLbiosynthesis genes (Figures 4 and 5). These results suggest that LJ angle might be sensitive to endogenous SL (Figure 7). In addition, D3 and D14, were up-regulated under $-\mathrm{P}$, indicating that sensitivity to endogenous SLs also increased under -P (Figure 5). Phosphate deficiency induces Syg1/Pho81/XPR1 (SPX1) and SPX2 expression, which inhibit REGULATOR OF LEAF INCLINATION (RLI1), a positive regulator of leaf inclination, reducing leaf angle via suppression of downstream BR signaling (Ruan et al., 2018). Under low N, leaf angle is reduced in rice and eucalyptus to avoid damage by photoinhibition (Close and Beadle, 2006; Kumagai et al., 2014). Under nutrient deficiencies, rice grows slowly and has fewer tillers, lower chlorophyll content, and lower yield (Dobermann and Fairhurst, 2001). The decrease in leaf chlorophyll content leads to production of reactive oxygen species, which attack cell 

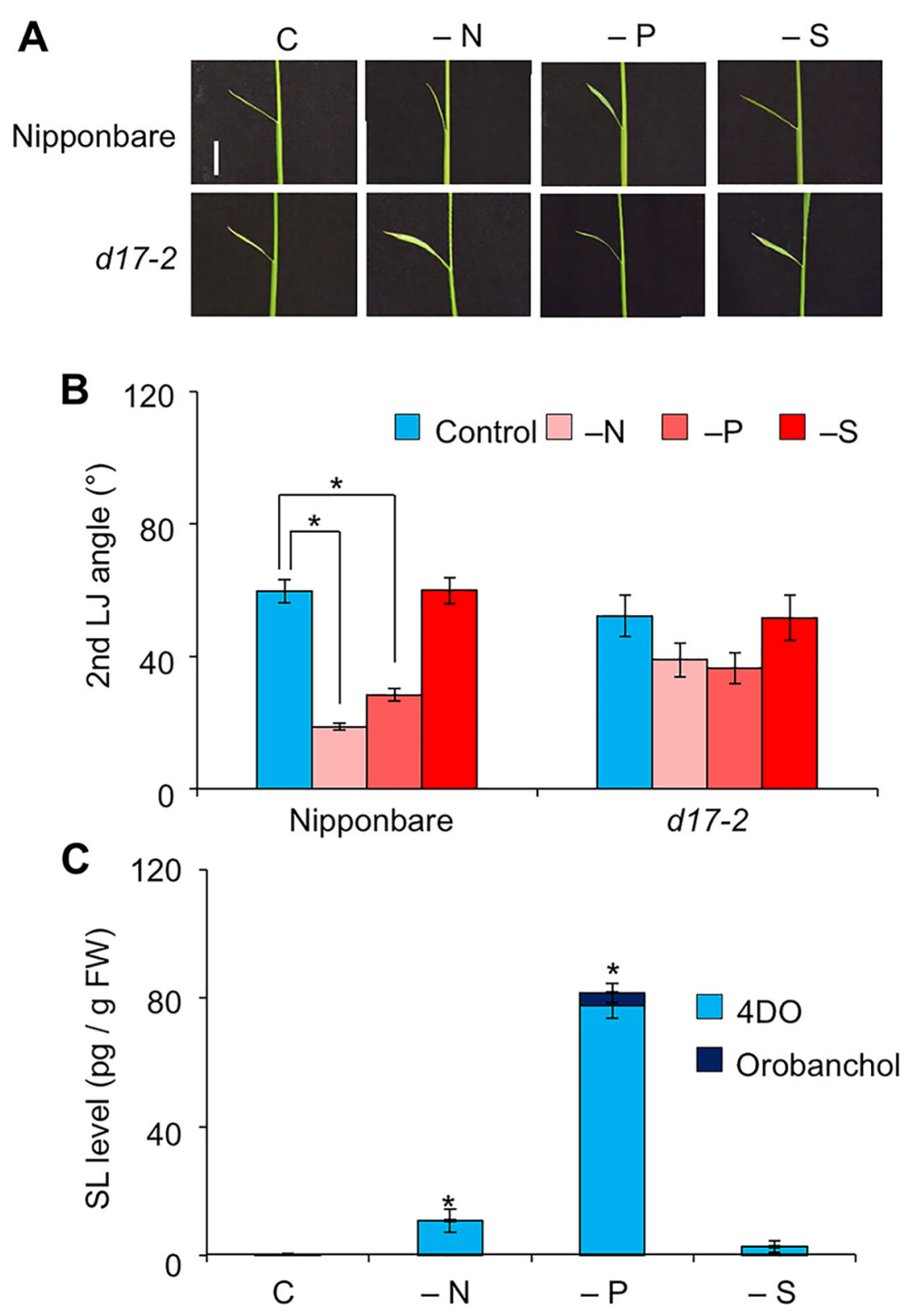

FIGURE 6 | Effect of macronutrient deficiency on LJ angle and SL levels in "Nipponbare"-background rice seedlings. (A) Images of the 2nd leaves. Bar, $1 \mathrm{~cm}$. (B) Second-LJ angle under macronutrient deficiencies. C, control. ${ }^{*} P<0.05$ (Student's $t$-test). Data are means $\pm S$.E. ( $n=5$ ). (C) SL levels in roots under nitrogen, phosphate, or sulfate deficiency in roots of 14 -d-old seedlings. C, control. ${ }^{\star} P<0.05$ (Student's $t$-test vs. control). Data are means \pm S.E. ( $n=4$ ).

components (Jia et al., 2003). High-intensity light causes photoinhibition of photosynthesis (Powles, 1984), although it also increases SL production in tomato roots (Koltai et al., 2011). Therefore, plants might decrease LJ angle to avoid photoinhibition via SL signals produced under $-\mathrm{N},-\mathrm{P}$, or $-\mathrm{S}$. Previously we suggested that plants use SL signaling to utilize limited nutrients efficiently and to adapt to poor nutrition because SLs are produced under $-\mathrm{N}$ and/or $-\mathrm{P}$ (Umehara, 2011). Here, we propose that SLs are important for photosynthetic efficiency and nutrient allocation.
We could not find rice canonical SLs, 4DO, or orobanchol, in the LJ even under $-\mathrm{P}$ when multiple SL genes were highly expressed (Figure 4, Supplementary Figure S3). In rice, SLs regulating the LJ angle might be non-canonical SLs such as carlactonoic acid and methyl carlactonoate (Yoneyama et al., 2018b). Canonical SLs might be transported from roots to the LJ and converted to active signaling molecules during the transportation, or induce other active signaling molecule.

Rice SL-related mutants of "Shiokari" background have increased LJ angle (Li et al., 2014). We found such phenotypes 


\section{Nutrient sufficient condition}

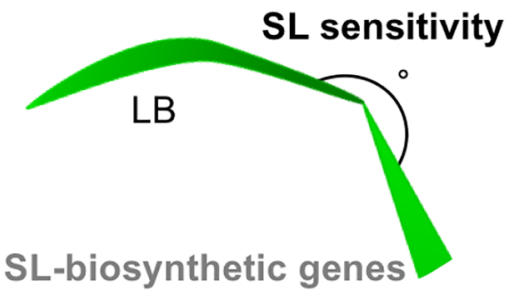

LS
$-\mathbf{N},-\mathrm{P},(-\mathrm{S})$

LB

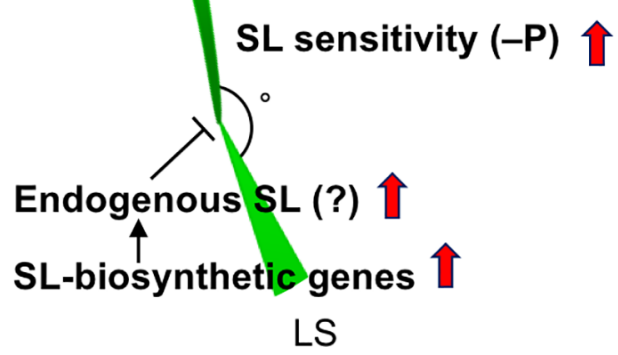

FIGURE 7 | A proposed model on LJ angle regulation through SL signals. Expression levels of SL-biosynthetic genes are low in nutrient sufficient condition, whereas they are up-regulated in $-\mathrm{N},-\mathrm{P}$ (-S in Shiokari), probably stimulating endogenous SL production in LJ. SL sensitivity of LJ is basically high, but the sensitivity is increased in -P. LB, leaf blade, LS, lead sheath.

in SL mutants in the "Shiokari" background (Figures 1 and 2). The phenotype of the SL-related mutants depends on the cultivar: the LJ angles in "Nipponbare", "Norin 8", and "Kasalath" were twice that of "Shiokari", with no significant differences between WT and SL mutants (Supplementary Figure S4). In response to $-\mathrm{N}$ and $-\mathrm{P}, \mathrm{SL}$ production was stimulated and the LJ angle decreased in "Nipponbare", but not in $d 17-2$ (Figure 6). This point was common between "Shiokari" and "Nipponbare". Thus, endogenous SL levels would increase in response to nutrient deficiencies and decrease leaf angle in rice. In the future, which types of SLs directly regulate the leaf angle would be clarified.

Auxin, GA, and BR also regulate LJ angle. The LJ angle was larger in SL mutants in the "Shiokari" background than in WT "Shiokari" (Figure 1), indicating that BR might accumulate in the LJ of the SL mutants or SL might positively regulate BR signaling. Interaction between SL and these phytohormones is well characterized in shoot branching regulation. In the BR signaling pathway, BRI-EMS-suppressor 1 (BES1) acts as a downstream transcription factor to positively regulate BRresponsive gene expression (Yin et al., 2002). In Arabidopsis, $\mathrm{BES} 1$ binds to MAX2, an ortholog of rice D3, and the complex is degraded to inhibit shoot branching (Wang et al., 2013). Auxin stimulates SL biosynthesis, whereas SL regulates polar auxin transport, contributing to shoot branching regulation (Domagalska and Leyser, 2011). Gibberellin inhibits SL biosynthesis in rice (Ito et al., 2017). The position of SL in this phytohormone cross-talk of LJ angle regulation remains unknown. Further analysis would be required to determine the interactions between SL and other phytohormones.

\section{DATA AVAILABILITY STATEMENT}

All datasets generated for this study are included in the article/ Supplementary Material.

\section{AUTHOR CONTRIBUTIONS}

MU and KS designed the research. MS and SY performed the experiments and analyzed the data. MS and MU wrote the paper.

\section{FUNDING}

This work was in part supported by a grant from Toyo University (the Inoue Enryo Memorial Foundation for Promoting Sciences to MS), from Ichimura Foundation for New Technology (the Plant Research Grant, No. 28-19 to MU), and from the Japan Society for the Promotion of Science (KAKENHI, No. 17K07650 to MU).

\section{ACKNOWLEDGMENTS}

We thank Prof. Junko Kyozuka (Tohoku University) for kindly providing seeds of "Shiokari", "Nipponbare", "Norin 8", and their SL mutants; Dr. Hiroaki Iwai (The University of Tsukuba) for kindly providing "Kasalath" seeds; Prof. Kohki Akiyama (Osaka Prefecture University) for kindly providing SL standards and $d_{1}$-labeled SLs; and Drs. Shosaku Kashiwada and Hiroki Higashibata (Toyo University) for constructive comments on our study. This study was also supported by the Research Center for Life and Environmental Sciences, Toyo University.

\section{SUPPLEMENTARY MATERIAL}

The Supplementary Material for this article can be found online at: https://www.frontiersin.org/articles/10.3389/fpls.2020.00135/ full\#supplementary-material 


\section{REFERENCES}

Agusti, J., Herold, S., Schwarz, M., Sanchez, P., Ljung, K., Dun, E. A., et al. (2011). Strigolactone signaling is required for auxin-dependent stimulation of secondary growth in plants. Proc. Natl. Acad. Sci. U.S.A. 108 (50), 2024220247. doi: 10.1073/pnas.1111902108

Akiyama, K., Matsuzaki, K., and Hayashi, H. (2005). Plant sesquiterpenes induce hyphal branching in arbuscular mycorrhizal fungi. Nature 435 (7043), 824827. doi: $10.1038 /$ nature 03608

Alder, A., Jamil, M., Marzorati, M., Bruno, M., Vermathen, M., Bigler, P., et al. (2012). The path from $\beta$-carotene to carlactone, a strigolactone-like plant hormone. Science 335 (6074), 1348-1351. doi: 10.1126/science.1218094

Arite, T., Iwata, H., Ohshima, K., Maekawa, M., Nakajima, M., Kojima, M., et al. (2007). DWARF10, an RMS1/MAX4/DAD1 ortholog, controls lateral bud outgrowth in rice. Plant J. 51 (6), 1019-1029. doi: 10.1111/j.1365313X.2007.03210.x

Arite, T., Umehara, M., Ishikawa, S., Hanada, A., Maekawa, M., Yamaguchi, S., et al. (2009). d14, a strigolactone-insensitive mutant of rice, shows an accelerated outgrowth of tillers. Plant Cell Physiol. 50 (8), 1416-1424. doi: 10.1093/pcp/pcp091

Bouwmeester, H. J., Roux, C., Lopez-Raez, J. A., and Becard, G. (2007). Rhizosphere communication of plants, parasitic plants and AM fungi. Trends Plant Sci. 12 (5), 224-230. doi: 10.1016/j.tplants.2007.03.009

Cao, H., and Chen, S. (1995). Brassinosteroid-induced rice lamina joint inclination and its relation to indole-3-acetic acid and ethylene. Plant Growth Regul. 16 (2), 189-196. doi: 10.1007/bf00029540

Close, D. C., and Beadle, C. L. (2006). Leaf angle responds to nitrogen supply in eucalypt seedlings. Is it a photoprotective mechanism? Tree Physiol. 26 (6), 743-748. doi: 10.1093/treephys/26.6.743

Cook, C. E., Whichard, L. P., Turner, B., Wall, M. E., and Egley, G. H. (1966). Germination of witchweed (Striga lutea Lour.): Isolation and properties of a potent stimulant. Science 154 (3753), 1189-1190. doi: 10.1126/ science.154.3753.1189

Cook, C. E., Whichard, L. P., Wall, M. E., Egley, G. H., Coggon, P., Luhan, P. A., et al. (1972). Germination stimulants. 2. The structure of strigol-a potent seed germination stmulant for witchweed (Striga lutea Lour.). J. Am. Chem. Soc. 94, 6198-6199. doi: 10.1021/ja00772a048

Dobermann, A., and Fairhurst, T. (2001). Rice: Nutrient Disorders \& Nutrient Management (Singapore and IRRI, Philippines: PPI/PPIC).

Domagalska, M. A., and Leyser, O. (2011). Signal integration in the control of shoot branching. Nat. Rev.: Mol. Cell Biol. 12 (4), 211-221. doi: 10.1038/ nrm3088

Gan, L., Wu, H., Wu, D., Zhang, Z., Guo, Z., Yang, N., et al. (2015). Methyl jasmonate inhibits lamina joint inclination by repressing brassinosteroid biosynthesis and signaling in rice. Plant Sci. 241, 238-245. doi: 10.1016/ j.plantsci.2015.10.012

Gomez-Roldan, V., Fermas, S., Brewer, P. B., Puech-Pages, V., Dun, E. A., Pillot, J. P., et al. (2008). Strigolactone inhibition of shoot branching. Nature 455 (7210), 189-194. doi: 10.1038/nature07271

Hasegawa, S., Tsutsumi, T., Fukushima, S., Okabe, Y., Saito, J., Katayama, M., et al. (2018). Low Infection of Phelipanche aegyptiaca in Micro-Tom Mutants Deficient in CAROTENOIDCLEAVAGE DIOXYGENASE 8. Int. J. Mol. Sci. 19 (9), e2645. doi: 10.3390/ijms19092645

Hoshikawa, K. (1989). The growing rice plant: An anatomical monograph (Japan: Nobunkyo).

Ishikawa, S., Maekawa, M., Arite, T., Onishi, K., Takamure, I., and Kyozuka, J. (2005). Suppression of tiller bud activity in tillering dwarf mutants of rice. Plant Cell Physiol. 46 (1), 79-86. doi: 10.1093/pcp/pci022

Ito, S., Yamagami, D., Umehara, M., Hanada, A., Yoshida, S., Sasaki, Y., et al. (2017). Regulation of strigolactone biosynthesis by gibberellin signaling. Plant Physiol. 174 (2), 1250-1259. doi: 10.1104/pp.17.00301

Jia, H. S., Han, Y. Q., and Li, D. Q. (2003). Photoinhibition and Active Oxygen Species Production in Detached Apple Leaves During Dehydration. Photosynthetica 41 (1), 151-156. doi: 10.1023/a:1025889219115

Jiang, L., Liu, X., Xiong, G., Liu, H., Chen, F., Wang, L., et al. (2013). DWARF 53 acts as a repressor of strigolactone signalling in rice. Nature 504 (7480), 401405. doi: $10.1038 /$ nature 12870
Kamachi, K., Yamaya, T., Mae, T., and Ojima, K. (1991). A role for glutaminesynthetase in the remobilization of leaf nitrogen during natural senescence in rice leaves. Plant Physiol. 96 (2), 411-417. doi: 10.1104/pp.96.2.411

Kapulnik, Y., Delaux, P. M., Resnick, N., Mayzlish-Gati, E., Wininger, S., Bhattacharya, C., et al. (2011). Strigolactones affect lateral root formation and root-hair elongation in Arabidopsis. Planta 233 (1), 209-216. doi: 10.1007/ s00425-010-1310-y

Kobae, Y., Kameoka, H., Sugimura, Y., Saito, K., Ohtomo, R., Fujiwara, T., et al. (2018). Strigolactone biosynthesis genes of rice is required for the punctual entry of arbuscular mycorrhizal fungi into the roots. Plant Cell Physiol. 59 (3), 544-553. doi: 10.1093/pcp/pcy001

Koltai, H., Cohen, M., Chesin, O., Mayzlish-Gati, E., Becard, G., Puech, V., et al. (2011). Light is a positive regulator of strigolactone levels in tomato roots. J. Plant Physiol. 168 (16), 1993-1996. doi: 10.1016/j.jplph.2011.05.022

Kumagai, E., Hamaoka, N., Araki, T., and Ueno, O. (2014). Dorsoventral asymmetry of photosynthesis and photoinhibition in flag leaves of two rice cultivars that differ in nitrogen response and leaf angle. Physiol. Plant. 151 (4), 533-543. doi: 10.1111/ppl.12145

Li, X., Sun, S., Li, C., Qiao, S., Wang, T., Leng, L., et al. (2014). The Strigolactonerelated mutants have enhanced lamina joint inclination phenotype at the seedling stage. J. Genet. Genomics 41 (11), 605-608. doi: 10.1016/ j.jgg.2014.09.004

Lin, H., Wang, R. X., Qian, Q., Yan, M. X., Meng, X. B., Fu, Z. M., et al. (2009). DWARF27, an iron-containing protein required for the biosynthesis of strigolactones, regulates rice tiller bud outgrowth. Plant Cell 21 (5), 15121525. doi: $10.1105 /$ tpc. 109.065987

Lopez-Raez, J. A., Charnikhova, T., Gomez-Roldan, V., Matusova, R., Kohlen, W., De Vos, R., et al. (2008). Tomato strigolactones are derived from carotenoids and their biosynthesis is promoted by phosphate starvation. New Phytol. 178, 863-874. doi: 10.1111/j.1469-8137.2008.02406.x

Mantilla-Perez, M. B., and Salas Fernandez, M. G. (2017). Differential manipulation of leaf angle throughout the canopy: current status and prospects. J. Exp. Bot. 68 (21-22), 5699-5717. doi: 10.1093/jxb/erx378

Nakamura, A., Fujioka, S., Takatsuto, S., Tsujimoto, M., Kitano, H., Yoshida, S., et al. (2009). Involvement of C-22-hydroxylated brassinosteroids in auxininduced lamina joint bending in rice. Plant Cell Physiol. 50 (9), 1627-1635. doi: $10.1093 /$ pcp/pcp106

Nelson, D. R., Schuler, M. A., Paquette, S. M., Werck-Reichhart, D., and Bak, S. (2004). Comparative genomics of rice and Arabidopsis. Analysis of 727 cytochrome P450 genes and pseudogenes from a monocot and a dicot. Plant Physiol. 135 (2), 756-772. doi: 10.1104/pp.104.039826

Powles, S. B. (1984). Photoinhibition of photosynthesis induced by visible light. Аnnu. Rev. Plant Physiol. 35 (1), 15-44. doi: 10.1146/annurev.pp.35.060184.000311

Ruan, W., Guo, M., Xu, L., Wang, X., Zhao, H., Wang, J., et al. (2018). An SPXRLI1 module regulates leaf inclination in response to phosphate availability in rice. Plant Cell 30 (4), 853-870. doi: 10.1105/tpc.17.00738

Ruyter-Spira, C., Kohlen, W., Charnikhova, T., van Zeijl, A., van Bezouwen, L., de Ruijter, N., et al. (2011). Physiological effects of the synthetic strigolactone analog GR24 on root system architecture in Arabidopsis: another belowground role for strigolactones? Plant Physiol. 155 (2), 721-734. doi: 10.1104/ pp. 110.166645

Sakamoto, T., Morinaka, Y., Ohnishi, T., Sunohara, H., Fujioka, S., UeguchiTanaka, M., et al. (2006). Erect leaves caused by brassinosteroid deficiency increase biomass production and grain yield in rice. Nat. Biotechnol. 24 (1), 105-109. doi: 10.1038/nbt1173

Schneider, C. A., Rasband, W. S., and Eliceiri, K. W. (2012). NIH Image to ImageJ: 25 years of image analysis. Nat. Methods 9 (7), 671-675. doi: 10.1038/ nmeth.2089

Seto, Y., Sado, A., Asami, K., Hanada, A., Umehara, M., Akiyama, K., et al. (2014). Carlactone is an endogenous biosynthetic precursor for strigolactones. Proc. Natl. Acad. Sci. U.S.A. 111 (4), 1640-1645. doi: 10.1073/pnas.1314805111

Seto, Y., Yasui, R., Kameoka, H., Tamiru, M., Cao, M., Terauchi, R., et al. (2019). Strigolactone perception and deactivation by a hydrolase receptor DWARF14. Nat. Commun. 10 (1), 191. doi: 10.1038/s41467-018-08124-7

Shimada, A., Ueguchi-Tanaka, M., Sakamoto, T., Fujioka, S., Takatsuto, S., Yoshida, S., et al. (2006). The rice SPINDLY gene functions as a negative regulator of gibberellin signaling by controlling the suppressive function of the 
DELLA protein, SLR1, and modulating brassinosteroid synthesis. Plant J. 48 (3), 390-402. doi: 10.1111/j.1365-313X.2006.02875.x

Shindo, M., Shimomura, K., Yamaguchi, S., and Umehara, M. (2018). Upregulation of DWARF27 is associated with increased strigolactone levels under sulfur deficiency in rice. Plant Direct 2 (4), e00050. doi: 10.1002/pld3.50

Sinclair, T. R., and Sheehy, J. E. (1999). Erect leaves and photosynthesis in rice. Science 283 (5407), 1455. doi: 10.1126/science.283.5407.1455c

Sun, H., Tao, J., Liu, S., Huang, S., Chen, S., Xie, X., et al. (2014). Strigolactones are involved in phosphate- and nitrate-deficiency-induced root development and auxin transport in rice. J. Exp. Bot. 65 (22), 6735-6746. doi: 10.1093/jxb/eru029

Sun, S., Chen, D., Li, X., Qiao, S., Shi, C., Li, C., et al. (2015). Brassinosteroid signaling regulates leaf erectness in Oryza sativa via the control of a specific $\mathrm{U}$ type cyclin and cell proliferation. Dev. Cell 34 (2), 220-228. doi: 10.1016/ j.devcel.2015.05.019

Tong, H., Xiao, Y., Liu, D., Gao, S., Liu, L., Yin, Y., et al. (2014). Brassinosteroid regulates cell elongation by modulating gibberellin metabolism in rice. Plant Cell 26 (11), 4376-4393. doi: 10.1105/tpc.114.132092

Ueda, H., and Kusaba, M. (2015). Strigolactone regulates leaf senescence in concert with ethylene in Arabidopsis. Plant Physiol. 169, 138-147. doi: 10.1104/pp.15.00325

Umehara, M., Hanada, A., Yoshida, S., Akiyama, K., Arite, T., Takeda-Kamiya, N., et al. (2008). Inhibition of shoot branching by new terpenoid plant hormones. Nature 455 (7210), 195-200. doi: 10.1038/nature07272

Umehara, M., Hanada, A., Magome, H., Takeda-Kamiya, N., and Yamaguchi, S. (2010). Contribution of strigolactones to the inhibition of tiller bud outgrowth under phosphate deficiency in rice. Plant Cell Physiol. 51 (7), 1118-1126. doi: $10.1093 / \mathrm{pcp} / \mathrm{pcq} 084$

Umehara, M. (2011). Strigolactone, a key regulator of nutrient allocation in plants. Plant Biotechnol. 28 (5), 429-437. doi: 10.5511/plantbiotechnology.11.1109a

Wada, K., Marumo, S., Ikekawa, N., Morisaki, M., and Mori, K. (1981). Brassinolide and homobrassinolide promotion of lamina inclination of rice seedlings. Plant Cell Physiol. 22 (2), 323-325. doi: 10.1093/ oxfordjournals.pcp.a076173

Wang, L., Wang, Z., Xu, Y., Joo, S. H., Kim, S. K., Xue, Z., et al. (2009). OsGSR1 is involved in crosstalk between gibberellins and brassinosteroids in rice. Plant J. 57 (3), 498-510. doi: 10.1111/j.1365-313X.2008.03707.x

Wang, Y., Sun, S., Zhu, W., Jia, K., Yang, H., and Wang, X. (2013). Strigolactone/ MAX2-induced degradation of brassinosteroid transcriptional effector BES1 regulates shoot branching. Dev. Cell 27 (6), 681-688. doi: 10.1016/ j.devcel.2013.11.010

Yamada, Y., Furusawa, S., Nagasaka, S., Shimomura, K., Yamaguchi, S., and Umehara, M. (2014). Strigolactone signaling regulates rice leaf senescence in response to a phosphate deficiency. Planta 240 (2), 399-408. doi: 10.1007/ s00425-014-2096-0

Yamada, Y., Otake, M., Furukawa, T., Shindo, M., Shimomura, K., Yamaguchi, S., et al. (2018). Effects of strigolactones on grain yield and seed development in rice. J. Plant Growth Regul. 38 (3), 753-764. doi: 10.1007/s00344-018-9887-7

Yin, Y., Wang, Z. Y., Mora-Garcia, S., Li, J., Yoshida, S., Asami, T., et al. (2002). BES1 accumulates in the nucleus in response to brassinosteroids to regulate gene expression and promote stem elongation. Cell 109 (2), 181-191. doi: 10.1016/s0092-8674(02)00721-3

Yoneyama, K., Xie, X., Kusumoto, D., Sekimoto, H., Sugimoto, Y., Takeuchi, Y., et al. (2007a). Nitrogen deficiency as well as phosphorus deficiency in sorghum promotes the production and exudation of 5-deoxystrigol, the host recognition signal for arbuscular mycorrhizal fungi and root parasites. Planta 227 (1), 125132. doi: $10.1007 / \mathrm{s} 00425-007-0600-5$

Yoneyama, K., Yoneyama, K., Takeuchi, Y., and Sekimoto, H. (2007b). Phosphorus deficiency in red clover promotes exudation of orobanchol, the signal for mycorrhizal symbionts and germination stimulant for root parasites. Planta 225 (4), 1031-1038. doi: 10.1007/s00425-006-0410-1

Yoneyama, K., Xie, X., Kim, H. I., Kisugi, T., Nomura, T., Sekimoto, H., et al. (2012). How do nitrogen and phosphorus deficiencies affect strigolactone production and exudation? Planta 235 (6), 1197-1207. doi: 10.1007/s00425011-1568-8

Yoneyama, K., Mori, N., Sato, T., Yoda, A., Xie, X., Okamoto, M., et al. (2018a). Conversion of carlactone to carlactonoic acid is a conserved function of MAX1 homologs in strigolactone biosynthesis. New Phytol. 218 (4), 1522-1533. doi: 10.1111/nph.15055

Yoneyama, K., Xie, X., Yoneyama, K., Kisugi, T., Nomura, T., Nakatani, Y., et al. (2018b). Which are the major players, canonical or non-canonical strigolactones? J. Exp. Bot. 69 (9), 2231-2239. doi: 10.1093/jxb/ery090

Zhang, L. Y., Bai, M. Y., Wu, J., Zhu, J. Y., Wang, H., Zhang, Z., et al. (2009). Antagonistic HLH/bHLH transcription factors mediate brassinosteroid regulation of cell elongation and plant development in rice and Arabidopsis. Plant Cell 21 (12), 3767-3780. doi: 10.1105/tpc.109.070441

Zhang, Y., van Dijk, A. D., Scaffidi, A., Flematti, G. R., Hofmann, M., Charnikhova, T., et al. (2014). Rice cytochrome P450 MAX1 homologs catalyze distinct steps in strigolactone biosynthesis. Nat. Chem. Biol. 10 (12), 1028-1033. doi: 10.1038/nchembio. 1660

Zhang, S., Wang, S., Xu, Y., Yu, C., Shen, C., Qian, Q., et al. (2015). The auxin response factor, OsARF19, controls rice leaf angles through positively regulating OsGH3-5 and OsBRI1. Plant Cell Environ. 38 (4), 638-654. doi: $10.1111 /$ pce. 12397

Zhao, S. Q., Hu, J., Guo, L. B., Qian, Q., and Xue, H. W. (2010). Rice leaf inclination2, a VIN3-like protein, regulates leaf angle through modulating cell division of the collar. Cell Res. 20 (8), 935-947. doi: 10.1038/cr.2010.109

Zhao, S. Q., Xiang, J. J., and Xue, H. W. (2013). Studies on the rice LEAF INCLINATION1 (LC1), an IAA-amido synthetase, reveal the effects of auxin in leaf inclination control. Mol. Plant 6 (1), 174-187. doi: 10.1093/mp/sss064

Zhou, F., Lin, Q., Zhu, L., Ren, Y., Zhou, K., Shabek, N., et al. (2013). D14-SCF (D3)-dependent degradation of D53 regulates strigolactone signalling. Nature 504 (7480), 406-410. doi: 10.1038/nature12878

Zou, J., Zhang, S., Zhang, W., Li, G., Chen, Z., Zhai, W., et al. (2006). The rice HIGH-TILLERING DWARF1 encoding an ortholog of Arabidopsis MAX3 is required for negative regulation of the outgrowth of axillary buds. Plant J. 48 (5), 687-698. doi: 10.1111/j.1365-313X.2006.02916.x

Conflict of Interest: The authors declare that the research was conducted in the absence of any commercial or financial relationships that could be construed as a potential conflict of interest.

Copyright (c) 2020 Shindo, Yamamoto, Shimomura and Umehara. This is an openaccess article distributed under the terms of the Creative Commons Attribution License (CC BY). The use, distribution or reproduction in other forums is permitted, provided the original author(s) and the copyright owner(s) are credited and that the original publication in this journal is cited, in accordance with accepted academic practice. No use, distribution or reproduction is permitted which does not comply with these terms. 